\title{
Comparativa de la Experiencia de Flip Teaching en dos asignaturas y centros distintos en la UPV
}

\author{
Oltra Gutierrez, Juan Vicente ${ }^{\mathrm{a}}$; Sofía Estelles-Miguel ${ }^{\mathrm{b}}$, Fernando Garrigos-Simon ${ }^{\mathrm{c}}$ y \\ Montesa Andrés, José Onofre ${ }^{d}$ \\ a,c,d Escuela Técnica Superior de Ingeniería Informática jvoltra@omp.upv.es; fgarrigos@doe.upv.es y \\ jomontes@omp.upv.es y b Facultad de Administración y Dirección de Empresas, \\ soesmi@omp.upv.es. Todos los autores del Departamento de Organización de Empresas de la \\ Universitat Politècnica de València.
}

\begin{abstract}
In this paper has been carried out a comparative of two different subjects, taught in two different degrees, both of them have been taught with the Flip Teaching methodology. Evidently, the specific characteristics of each of them have been taken into account. The subjects, degrees and school in which the experience has been realized are: Deontology and Professionalism in Computer Engineering degree in the School of Informatics and Operations Management in the Business Administration and Management in the Faculty of Business Administration and Management. Both subject are taught by professors of Deparment of Business Organization. The experience began in the academic year 2014/2015 and has lasted until the current 2016/2017.
\end{abstract}

Keywords: Flip-Teaching, High Education, Deontology, Operations Management.

\footnotetext{
Resumen

En el presente trabajo se ha llevado a cabo una comparativa entre dos asignaturas diferentes, impartidas en dos grados distintos, pero donde ambas han sido impartidas utilizando la metodología de Flip Teaching o Docencia Inversa. Evidentemente, se han tenido en cuenta las características especificas de cada una de ellas. Las asignaturas, titulaciones y cursos en los se que ha realizado la experiencia son: Deontología y Profesionalismo en el grado de Ingeniería Informática en la Escuela Técnica Superior de Ingeniería Informática y Dirección de Producción y Operaciones en el Grado de Administración y Dirección de Empresas en la Facultad del mismo nombre. Ambas materias son impartidas por profesores del Departamento de Organización de Empresas. La experiencia comenzó en el curso 2014/2015 y se ha extendido hasta el actual 2016/2017.
}

Palabras clave: Flip Teaching, Docencia Inversa, Educación Universitaria, Deontología, Dirección de Operaciones. 


\section{Introducción}

El Vicerrectorado de Tecnologías de la Información y de las Comunicaciones (VTIC) de la Universitat Politècnica de València (UPV), inició la experiencia de "Flip Teaching" o Docencia Inversa durante el curso 2014-2015. La misma se realizó en los segundos cursos de los grados de Administración y Dirección de Empresas e Ingeniería Informática. Se planteó en los siguientes términos (UPV, 2014):

- Las clases presenciales teóricas (teoría de aula) o, al menos, un porcentaje elevado de éstas se elaborarán, por parte de los profesores de la asignatura, como materiales audiovisuales de alta calidad.

- Los alumnos que participen en está metodología se estudiarán los conceptos teóricos visualizando estos materiales y consultando el material docente que el profesor estime oportuno de forma no presencial.

- Los grupos en los que se realice la experiencia serán reducidos, de 20 a 30 alumnos. Siendo el criterio de acceso el orden de matrícula establecido en la normativa académica UPV.

- El tiempo dedicado por parte del alumno a actividades presenciales disminuirá en valor absoluto (dependiendo de la cantidad de teoría de aula que se elabore de forma audiovisual).

- Existirá una interacción bidireccional profesor/alumno en el proceso de aprendizaje. Las horas presenciales se dedicarán a la realización de actividades de consolidación de los conceptos teóricos y en una atención personalizada por parte del profesor para resolver las dudas que se planteen y una actitud proactiva del alumno en el aula.

En base a los parámetros arriba indicados y que habían sido establecidos por el VTIC (remarcando que la docencia inversa tiene como objetivo aprender metodologías docentes que nos permitan integrar en la docencia presencial contenidos docentes digitales utilizando las tecnologías que hoy en día tenemos a nuestro alcance (Botti, 2014)), las distintas asignaturas rediseñaron sus estructuras, de forma que la forma de impartición y la evaluación casaran con el formato conocido como FlipTeaching o de Docencia Inversa. (Bennett et al, 2011) y (Oltra, 2015).

Otro cambio importante introducido por la adaptación al Espacio Europeo de Educación Superior (EEES) es centrar la atención en el desarrollo de competencias y habilidades que preparan a los alumnos para su integración en el mundo laboral (Estellés et al, 2016). Partiendo de la base de la necesidad de cambiar la dinámica de las clases tradicionales basadas en el aprendizaje memorístico de conocimientos, en otra donde los estudiantes sean una parte activa, pasando de ser sujetos pasivos a ser protagonistas y responsables de su propio aprendizaje (Zabala y Arnau, 2007). Además de ayudar a que el alumno aprenda a su ritmo y permite al profesor atender las necesidades específicas de cada alumno (EstellesMiguel, et al, 2015).

El Departamento de Organización de Empresas, es transversal en la universidad, lo que ha permitido impartir este modelo de docencia en las dos titulaciones piloto. Sin embargo, en

(c)) BY-NC-ND 2017, Universitat Politècnica de València 
su momento se consideró que sería un error aplicar los mismos esquemas y organización de evaluaciones para alumnos con procedencia y fin tan dispar.

La personalización de la educación para los estudiantes, produce múltiples beneficios en relación con el rendimiento académico. A la vez que mejora la posibilidad de que muchos estudiantes continúen estudiando, desarrolla mayor responsabilidad, ayuda en el entendimiento por encima de la pura memorización y desarrolla habilidades como la colaboración, la comunicación y la resolución de problemas (Vasileva et al, 2015). Además de mejorar la participación de los alumnos y aumentar la interacción entre estudiantes y profesores (Bethany, 2012).

Por supuesto para ello se necesita un cambio de rol por parte del profesor, acostumbrado a la docencia con clases magistrales en aula. De esta forma el profesor ayuda a los alumnos a desarrollar sus conocimientos y competencias (Tedesco, 2010). A la vez que se hace imprescindible la participación activa del alumno que no puede tomar una actitud pasiva en la clase sino todo lo contrario.

En el presente trabajo se presentan las dos experiencias en cada uno de los centros piloto, en la Escuela Técnica Superior de Ingeniería Informática (ETSInf) y en la Facultad de Administración y Dirección de Empresas (FADE), ambas de la Universitat Politècnica de Valencia, con las divergencias esperables sobre tipo de alumno y contenidos. Siendo la principal convergencia la metodología y el departamento al que pertenecen los profesores. Las asignaturas en las que se desarrollo la experiencia han sido Deontología y Profesionalismo en ETSInf y Dirección de Producción y Operaciones en FADE, ambas impartidas en segundo curso de grado.

\section{Dirección de Producción y Operaciones}

Esta asignatura es troncal obligatoria de 6 créditos, ubicada en el primer cuatrimestre del segundo curso de Grado de Administración y Dirección de Empresas en FADE de la UPV. $\mathrm{Su}$ contenido se fundamenta sobre la forma en que las organizaciones producen bienes $\mathrm{y}$ servicios. En ella se explica que cada objeto que se utiliza en la vida cotidiana ó cada tratamiento o servicio que se recibe por ejemplo en un banco, en una agencia de viajes o en una peluquería, incluso cada clase que se imparte en la universidad llegan a nosotros gracias a los Directores de Operaciones, que son los que gestionan la producción de los mismos. Con esta asignatura se pretende que los alumnos adquieran conocimientos específicos sobre las características fundamentales de la Dirección de Operaciones en las empresas (Albarracín et al., 2013). Es eminentemente práctica, aunque recoge también la explicación de algunos conceptos básicos para la realización de la parte práctica.

Las horas de impartición de la asignatura se distribuyen de la siguiente manera:

- 30 horas de teoría de aula:

- 10 horas de diseño del sistema de operaciones.

- 15 horas de sistema de planificación y control de operaciones.

- 5 horas de sistema logístico.

- 15 horas de prácticas de aula: 
- 3 horas de diseño del sistema de operaciones.

- 10 horas de sistema de planificación y control de operaciones.

- 2 horas de sistema logístico.

- 15 horas de prácticas de laboratorio:

- 3 horas de diseño del sistema de operaciones.

- 9 horas de sistema de planificación y control de operaciones.

- 3 horas de sistema logístico.

La asignatura se presenta mediante lecciones magistrales a los alumnos en las que se explica la teoría y a continuación se realizan problemas aplicados a los contenidos explicados. Además, los alumnos elaboran un aprendizaje basado en proyectos que se recoge en un portafolio en el que desarrollan el diseño del sistema productivo de una empresa, en el mismo, diseñan el producto, diseñan el proceso, finalmente realizan la distribución en planta de dicho proceso y finalmente deciden su localización.

La evaluación consiste en una prueba escrita de respuesta abierta que supone el $40 \%$ del peso de la nota, dos pruebas objetivas que suponen el $30 \%$ entre las dos, un portafolio (comentado en el párrafo anterior) que supone el 15\% y un caso que supone el 15\% del peso de la nota. No existen notas mínimas en ninguna de las partes para aprobar y entre todas las notas hay que llegar a una nota mínima de 5 .

\section{Deontología y Profesionalismo}

Es una asignatura troncal obligatoria de 4,5 créditos ( 3 en su parte teórica, 1,5 en su parte práctica), impartida durante el segundo semestre del curso segundo del Grado de Informática ETSInf de la UPV.

La asignatura tiene unas características que la hacen distinta al resto, como se puede apreciar dando un vistazo a los nombres del resto de asignaturas que comparten semestre: Redes de Computadores, Estructura de computadores, Concurrencia y sistemas distribuidos, Estructuras de datos y algoritmos e Interfaces persona computador. Esto permite con una rápida apreciación intuitiva, que resulta confirmada por la realidad, hacerse una idea aproximada de lo que el alumno ve en el resto de sus asignaturas. Que es una practicidad inmediata, mientras que en ésta, donde se les habla de las buenas prácticas y la deontología profesional, además de darles una visión del marco legal en el que se van a mover, resultará a priori para su percepción algo más apartado de su día a día, lo que implica un mayor esfuerzo del docente para lograr su implicación.

Se intenta acercar al alumno la legislación más importante que afecta a su desarrollo profesional (Propiedad intelectual -tipos de licencias-, Protección de datos, Código penal, leyes, reglamentos y otras). A los aspectos deontológicos y éticos de la profesión y el estudio de los códigos éticos más significativos, se añade el análisis del profesionalismo y competencias en el ámbito de las Tecnologías de la Información (TI) y una visión del asociacionismo y colegios profesionales.

Los contenidos se dividen en tres categorías, tras un tema introductorio: Profesionalismo Informático, donde ven conceptos básicos de profesionalismo e historia de la profesión

(cc)) EY-NC-ND 2017, Universitat Politècnica de València 
informática, y relacionados con el profesional en la organización y fuera de ella. Posteriormente se ven Aspectos Legales en la Informática, donde partiendo de conceptos básicos y marco legal de la actividad del profesional informático, se pone acento en protección de datos, propiedad intelectual y pruebas periciales informáticas. Finalmente, una tercera parte, Deontología Informática, donde partiendo de conceptos básicos se llega a las particularidades de la deontología informática: lo qué nos diferencia de otras profesiones.

La evaluación de la misma en su modalidad ordinaria -no FLIP- se realiza mediante boletines de prácticas (cuatro entregas, con un peso total de 3,5 puntos), tres casos de aula con un peso total de 1,5 puntos y dos parciales de 2,5 puntos cada uno, totalizando así los 10 puntos de la asignatura. Además, se les proponen distintas actividades y trabajos que les sirven para subir nota y, en cierto modo, para recuperar alguna actividad que no hubiese sido superada.

\section{Adaptación al Flip Teaching}

\subsection{Dirección de Producción y Operaciones}

Para la conversión de la asignatura en Docencia Inversa se ha intentado combinar las mejores prácticas de la formación tradicional y del enfoque on-line, intentando orientarlo de forma que el aprendizaje sea el adecuado para el estudiante. Hay que tener en cuenta las necesidades de aprendizaje de los estudiantes actuales consumidores masivos de pantallas y no tanto de papel (Vasileva et al, 2015). Al proporcionar un ambiente adaptativo a las necesidades del estudiante, les ayudamos a conseguir un rendimiento óptimo. Para ello es necesaria una cuidadosa planificación del trabajo a realizar por el alumno.

Con la utilización de las nuevas tecnologías un estudiante puede ver u oír una y otra vez aquello que no le quede claro, o que quiera volver a repasar, ir a su propio ritmo, pudiendo recuperar esa clase a la que no pudieron asistir (Sams y Bergmann, 2013). Indicar que las grabaciones se pusieron a disposición tanto de los alumnos del grupo Flip Teaching como del resto. Aunque el primer año sólo las utilizaron los alumnos del grupo Docencia Inversa, en el siguiente año se ha ido incrementando la visualización de los vídeos por parte de todos los alumnos de la asignatura con el fin de aclarar conceptos.

La UPV dispone de la plataforma Poliformat (basada en Sakai) que permite muchas posibilidades: colgar recursos de distintos tipos, enlaces, uso de foros, exámenes online, cuestionarios, programación de tareas, uso de chat y correo interno... Además también posee estudios de grabación que favorecen la elaboración de vídeos multimedia (polimedias), dentro de estos estarían los objetos de aprendizaje que deben cumplir unos requisitos determinados como estar descontextualizados y tener una duración inferior a 10 minutos (Estelles et al, 2014).

Con la implantación de la nueva metodología, la parte de teoría de aula se "enlato" al máximo (70\%) con vídeo-apuntes, objetos de formación y documentos en formato texto. 
Estos se clasificaron y ordenaron el apartado contenidos, con el fin de facilitar al máximo el seguimiento de los mismos por parte del alumno. Para la parte de teoría de aula, todos los temas se pusieron a disposición del alumno en formato testo, de cada tema había como mínimo un vídeo que explicaba lo esencial del tema. Además había una fecha de examen tipo test por Poliformat para cada tema, el alumno tenía todo un día para poder realizar el examen pero una vez lo iniciaba tenía un tiempo límite que se había establecido en el programa. Aún así se dejó una clase de dos horas por semana para hacer un seguimiento de los alumnos, explicarles los conceptos que se veía a través de los test que no estaban tan claros o para consultas o tutorías. Las prácticas de laboratorio se realizaron de la misma forma que para el resto de grupos. Sin embargo la parte de prácticas de aula, se "enlato" un $40 \%$, utilizando video-apuntes, de problemas grabados, realizados en pizarra, además los alumnos disponían de una extensa colección de problemas resueltos que se ponen a su disposición a través de la plataforma.

\subsection{Deontología y Profesionalismo}

Se imparten en ella temas, como hemos visto antes, con suficiente disimilitud con el resto de las asignaturas como para que resulte al docente un reto el lograr su homologación con ellas en el imaginario colectivo del alumnado. En la otra parte de la balanza, tenemos el hecho de que se trate de una población con no solo altos conocimientos, sino también fuerte interés en las TIC. Lo que nos facilita el uso de recursos que en otras titulaciones no tendrían un éxito claro, más allá del mero visionado de vídeos. Estos alumnos tienen tecnología líquida en las venas, así que piden mucho más.

En la experiencia, la carga práctica (laboratorio) quedó igual que en el resto de los grupos. Pero la teoría sufrió un cambio radical.

Así, todo el material de teoría se encuentra en la plataforma Poliformat, en su sección de contenidos, que, apoyándonos en el lenguaje html, permitió diseñar una hoja de ruta para cada tema donde se intercalaban los apuntes en formato pdf, (se quiera o no, imprescindibles para un conocimiento exhaustivo de la materia) con las clases grabadas en formato vídeo, en esas píldoras llamadas polimedias que se han citado, además de enlaces a documentales que pueden servir de apoyo, vídeos de youtube relacionados y otro material. A destacar una sección de "conceptos" grabados como audio, para que los alumnos puedan usar de esos ficheros mp3 mientras hacen deporte, esperan al tranvía, etc. También cada tema consta de una sección titulada "Para saber más", con documentos adicionales para el alumno curioso.

Esto queda plasmado en el siguiente esquema:

- Usamos material que nos permita sustituir la clase directa, de forma que al favorecer que los alumnos la puedan seguir en casa, trasladamos ese tiempo de trabajo allí, liberándonos el tiempo de aula para esas actividades de refuerzo que asumimos deben realizar por su cuenta y que cuya realización no siempre podemos constatar. Además, como nos indica (Sams y Bergmann, 2013) podemos usar ese tiempo para ayudar a los estudiantes que no llegan, con material de apoyo

(cc) EY-NC-ND 2017, Universitat Politècnica de València 
complementario, y lanzar desafíos a aquellos que ya han dominado el contenido, facilitándoles otro tipo de material que de una forma un tanto coloquial, bautizamos con la etiqueta "Para saber más". Del total de sesiones, una tercera parte se dedican a una tutoría presencial pura y dura, de manera que el alumno que no la precisa, no tiene porqué acudir. Los que si vienen son los que se encuentran atascados con alguna de las tareas a realizar en el curso o con algún concepto teórico que precise una mayor aclaración.

- Por otra parte, siguiendo el ejemplo de (Roshan, 2011) tratamos de romper otra atadura: la que los liga a un ordenador, sea portátil o de sobremesa. Para ello preparamos material que puede ser consumido también desde un smartphone o una tableta, y así arañaremos tiempo de una mina que suele estar sin explotar: el que habitualmente se pierde en el metro, el autobús... buscando la conversión de los transportes públicos en pequeñas aulas portátiles.

La diferencia fundamental que existe, y provocamos, con los materiales clásicos está en su adaptabilidad. Un estudiante puede volver a recibir una clase, como repaso antes del examen, escuchar de nuevo aquellos conceptos que le queden confusos y, no lo perdamos de vista, todo a su propio ritmo, pues no hay dos alumnos iguales, y el tiempo parecer ir a distinta velocidad en unos casos u otros. Recuperar clases perdidas por enfermedad se hace más fácil y, algo casi imposible, el lograr la concentración en el aula para asimilar conceptos complejos, resulta fácil al poder trasladar el aula su propia casa (Roshan, 2011).

A la hora de seleccionar las herramientas a emplear, el punto de partida necesario es revisar que herramientas pone a nuestra disposición la Universitat Politècnica de València. Centrándonos en la plataforma Poliformat antes citada (basada en Sakai), con sus muchas posibilidades, algunas elementales y otras de mayor complejidad, como colgar recursos de distintos tipos, almacenar enlaces, uso de foros, exámenes online, cuestionarios, programación de tareas, uso de chat y correo interno... Conviene destacar desde éste primer momento a una de éstas herramientas en concreto, Contenidos, con la que se pueden elaborar guías que ayuden a los alumnos a descubrir los materiales y a usar el resto de herramientas y funcionalidades de Poliformat en el orden adecuado y en el plazo correspondiente.

Si se añade a esto la posibilidad de generar contenidos de calidad, gracias a los estudios de grabación de la universidad que favorecen la creación de vídeos docentes (llamados polimedias), de unos 10 minutos de duración.

Para cubrir la totalidad de la asignatura, se usan una gran diversidad de elementos digitales distintos:

- Ficheros con formato pdf:

- Apuntes de la asignatura, en su versión clásica. Para su estudio reposado, mediante su impresión, envío a reprografía o uso de una pantalla. Aunque de una u otra manera sus contenidos resulten redundantes al encontrarse también repartidos en el resto de contenidos, siempre son un elemento de interés para el alumno. 
- Guiones de trabajo para casos de aula o prácticas de laboratorio. Instrucciones precisas para trabajar en el aula como seminario, con adaptaciones de casos reales, o para guía de trabajo durante las prácticas.

- Artículos, documentos de trabajo (normas y códigos) o libros con licencia "creative commons", sirven para actividades de refuerzo, o para ayuda al alumno inquieto que quiere "saber más". Siempre identificables como tales.

- $\quad$ Audio

- Ficheros en formato $\mathrm{mp} 3$, con resúmenes, definiciones o fragmentos muy breves de teoría, preparados para apoyar actividades de repaso, con plena movilidad del estudiante, que no dependerá así de una pantalla.

- Videos:

- Documentales, con contenido de interés, con licencia "creative commons" o abiertos en la plataforma de su propietario (generalmente documentales de RTVE). Otros vídeos docentes, en plataformas como youtube. Sirven de apoyo a los casos planteados, o para ampliación de conocimientos.

- Capturas de pantalla (screencast) y los citados polimedias, vídeos con contenido docente. La creación de éstos y otros materiales con distinto software e instalaciones de apoyo, quedan fuera del ámbito de la presente comunicación, pero resulta conveniente siquiera dejar la idea de que, al margen de los medios que proporciona la universidad, existe una gran cantidad de software libre, con licencias freeware, creativecommons, etc., muy útil para tal fin.

- Actos académicos grabados. Cada año se trae a conferenciantes afines a los contenidos de la materia. Sus conferencias se graban y están disponibles para su visionado, durante el año académico en la plataforma Poliformat, y a partir de entonces, en Youtube.

Estos elementos por si solos no son útiles si no se introducen en el proceso de comunicación con los alumnos, poniéndolos en el orden adecuado y estableciendo mecanismos de retroalimentación para evaluar su impacto y para calificarlos.

La herramienta principal que se usa para ordenarlos, y que de paso sirve de nexo de unión, conduciendo con textos breves de unos a otros e introduciéndolos, es "Contenidos", de PoliformaT. La herramienta "Contenidos" es un editor html donde se puede introducir texto, imágenes y enlaces a los elementos que antes se han citado, permitiendo de esta forma dar paso a sencillos tests de comprensión sin que el alumno abandone su navegación, y así se convierte en algo intuitivo.

Cuando los alumnos disponen del material necesario, pautado en tiempos, se hace necesario que el profesor no quede a ciegas, sin saber si van digiriendo la información en su poder, más allá de la clásica evaluación mediante un examen en papel, que reviste el peligro de llegar tarde para enmendar problemas que puedan ir surgiendo.

Para conseguir este fin, se emplean diferentes herramientas que superan al examen permitiendo un uso asíncrono de las mismas, en el que docente no tienen por qué coincidir.

(c)) BY-NC-ND 2017, Universitat Politècnica de València 
Algunas, como los "Foros", requieren de un seguimiento en tiempo. En la actualidad, estos foros son de acceso libre y voluntario, pero las mejores participaciones se premian (uso de la ludificación). Otros, como los "Encuestas" ó "Exámenes" online, permiten distintos grados de automatización. Todo ello sin perder de vista que para las entregas de pequeños trabajos o resultados de prácticas, mediante la programación de "Tareas" de esta forma el camino del alumno queda balizado, y además también la corrección del profesor, se agiliza mucho.

La evaluación pasa por trabajo en aula y casa. Los dos parciales, que sumaban 5 puntos, se sustituyen por cinco casos de aula y/o tareas a entregar, además de los tres casos de aula de 0,5 puntos que el resto de sus compañeros realizan.

De forma adicional se incluyen actividades de gamificación, en las que se premian las mejores intervenciones en debates, en los foros y en Twitter. En este último se hace un seguimiento de las noticias destacadas relacionadas con la asignatura.

Los alumnos también pueden optar a realizar un trabajo basado en la creación de un vídeo de 5 minutos sobre un tema propuesto, con dos premisas: el profesor revisará el guión antes de grabarlo y dará el material teórico necesario para construirlo.

Cuando se establecieron los cambios para la modalidad Flip Teaching, se mantuvieron los exámenes convencionales como una opción a la que el alumno podía optar. Cabe decir que no la eligió ninguno de ellos.

Las notas se descomponen en partes muy pequeñas. Eso implica que en el caso de no realizar, o fracasar en unas pocas, la continuidad en la asignatura no se resiente. Se intenta así evitar el abandono, al enganchar a los discentes en la sucesión, como un goteo, no molesto por su pequeño tamaño, pero persistente. Los casos permiten "tocar la realidad", de forma que la aplicación de los conocimientos teóricos es inmediata.

\section{Resultados}

En el presente apartado se habla de los resultados obtenidos en ambas asignaturas. La encuesta que se realizó fue la misma.

De las encuentas efectuadas a los alumnos se ha podido ver que la mayoría no eligió la modalidad Flip Teaching por su metodología, sino por incompatibilidad de horarios, o por tener un horario de clase presencial más reducido y poder gestionar mejor su tiempo.

Hay distintos elementos de medición aplicables a la docencia en general (Andrés, 2005) que resultan de interés por permitirnos comparativas entre grupos.

Porcentaje de Asistencia del alumnado. Con éste indicador se alude al porcentaje de alumnos que no han faltado más del $20 \%$ de las horas lectivas. El dato viene condicionado por la indicación, en la guía docente de la asignatura, de una asistencia mínima a un $80 \%$ de la asignatura. Dado que se reducen las horas lectivas, también las ausencias declinan su presencia. El dato se expresa como la división entre el número de alumnos que cumplen la condición de asistencia y el número total de alumnos, multiplicado por cien. En nuestro caso la tasa es de un $100 \%$. Para el resto de alumnos, el dato es de un $90 \%$. 
Porcentaje de aprobados: clásico indicador de rendimiento global, calculado como el número de alumnos con una nota igual o superior a 5, dividido por el total de matriculados, multiplicado por 100. En el caso de los alumnos que cursan por Flip Teaching, al ir generando pequeñas calificaciones durante el curso, el dato es ya de un $100 \%$ de éxito. Ningún alumno ha suspendido la asignatura. Con respecto a la asignatura impartida en ADE, se constata una subida de la nota media en un $25 \%$ con respecto a los alumnos que no optaron por esta modalidad.

Al margen de éstos indicadores, hay otros elementos que pasan por la percepción del alumnado, y que sin dejar de ser muy interesantes para verificar el interés conseguido por la experiencia y el grado de aceptación de la materia en éste formato, son imposibles de captar sin su participación activa. Para éste fin, se ha diseñado un cuestionario, inspirado entre otros por (Bethany, 2012), para ser respondido por los alumnos al fin de la misma.

Los elementos que la componen oscilan en torno a puntos como:

- La utilidad de los contenidos, desde la percepción del alumnado.

- La satisfacción de los alumnos tanto con los contenidos como con las herramientas empleadas y con el propio profesor.

- Los tiempos invertidos realmente por ellos, como comparativo de los cálculos previos realizados en la planificación: número de horas dedicadas, carga de trabajo con respecto a otras materias...

- Observaciones que los alumnos quieran aportar al proceso.

\subsection{Respuestas más Significativas}

\subsubsection{Modelo de Evaluación}

Se les pregunta sobre su preferencia entre el modelo clásico, con exámenes y un número reducido de casos de aula, frente al propuesto, basado en casos y tareas. Esto se les pregunta tanto al principio de la asignatura, para conocer su postura y obrar en consecuencia, como al final, cuando ya conocen con detalle la ruta que se ha seguido.

La primera respuesta la tenemos, obviamente, tanto para el curso anterior como para el presente. Y el resultado es virtualmente idéntico, situándose en un $90 \%$. La respuesta al final del curso anterior cambió, situando en un $100 \%$ la cifra de alumnos que preferían el modelo seguido al clásico.

Puede parecer que esta información no es relevante para lo que nos ocupa, pero lo es, y mucho: el modelo clásico les da a los alumnos unos momentos de tensión máxima, los próximos a las pruebas, y largos periodos de relajación, mientras que de la manera en que se trabaja, mantienen en todo momento un nivel bajo de trabajo, equivalente a la división de los créditos ECTS de la asignatura en su tiempo de desarrollo.

\subsubsection{Uso de la Herramienta "Foros"}

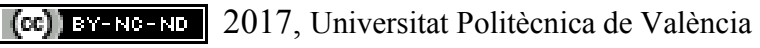


En este caso tenemos datos sobre el uso de los foros. Sobre el conocimiento de los mismos y el uso previo, los datos son también parecidos en ambas ediciones: un $90 \%$ de ellos han sido usuarios de foros, aunque solo un $10 \%$ de ellos los han usado dentro de Poliformat.

Su uso es algo voluntario, aunque los foros de "FAQ" (de ayuda, apoyo a las tutorías) se han incrementado, dando respuestas no solo sobre la teoría de la asignatura, sino a entregas, mecánicas del Flip Teaching, etc.

\subsubsection{Utilización del Material en Formato Texto}

Las respuestas obtenidas indicaban que no había mayor problema con este tipo de material, pero en algunos casos preferían documentales a tener que leer. Estos alumnos son muy visuales y las nuevas tecnologías hacen que los profesores al final nos tengamos que ir adaptando a ellas.

\subsubsection{Tiempo Invertido por el Alumno}

Cuando se prepararon los materiales, se hizo teniendo muy en cuenta la inversión de tiempo que los alumnos tendrían que hacer en casa. Ese cálculo incluía la visualización, lectura, audición... etc. de los materiales, con un tiempo holgado para su correcta comprensión.

Al analizar los datos, comprobamos que con la excepción del material depositado en formato texto, los tiempos empleados quedaban muy por debajo de los cálculos previos. Sin embargo, en lo que respecta a los ficheros de texto, sus cifras eran prácticamente las del profesor, indicando que si bien el cálculo era acertado en este aspecto, se habían ponderado mal en relación al resto de materiales.

\section{Conclusiones}

Con respecto a la aplicación del modelo de docencia inversa también conocido como Flip Teaching en la asignatura de Dirección de Producción y Operaciones en $2^{\circ}$ curso de FADE, cabe destacar que en esta asignatura se ha "enlatado" un 70\% de la teoría de aula y un $40 \%$ de las prácticas de aula y se ha mantenido el método de evaluación, se han incorporado exámenes tipo test para realizar un seguimiento de los conocimientos adquiridos por los alumnos. Todo ello, ha representado un importante esfuerzo tanto de planificación como de trabajo previo (conversión de material, edición, grabaciones, y preparación de material adicional) por parte de los profesores. No es fácil la creación de este tipo de materiales e incluso el tomar la decisión de qué parte pasar a este tipo de formato. De igual manera, ha representado un esfuerzo importante para los alumnos a la hora de adaptarse a esta nueva forma de trabajo. Pero la mayor parte de ellos lo han valorado como una experiencia positiva.

Con respecto a la asignatura Deontología y Profesionalismo, en $2^{\circ}$ curso del grado de informática, cabe destacar que de los elementos que se suelen considerar a la hora de medir la docencia en general, tanto porcentaje de asistencia, el de trabajos entregados en plazo, como el de aprobados (considerando las pruebas realizadas y evaluadas hasta la fecha), los datos son magníficos, superando las perspectivas iniciales, que venían matizadas por la inclusión de un grupo grande con alumnos que no se habían presentado voluntarios a la experiencia. En cuanto al porcentaje de contenidos impartidos, siendo este expresado como 
los contenidos impartidos, dividido por el total contenidos programados, multiplicado por cien, cumplimos a rajatabla con el calendario previsto que, por otra parte, supone una semana de adelanto con respecto a los grupos no Flip Teaching, permitiéndonos reservar el tiempo sobrante al final para dedicarlo a actividades de seminario y prácticas. Quedaría por último un factor puramente cualitativo, de muy difícil medición, por lo personal: la satisfacción del docente. Y solo cabe decir que ésta es muy elevada, dando la sensación, en expresión manida, del "deber cumplido".

\section{Referencias}

ALBARRACIN GUILLEM, J.M., ESTELLES-MIGUEL, SOFIA y BABILONI, E. (2013). Guía Docente de la Asignatura de Dirección de Producción y Operaciones. Disponible en: https://poliformat.upv.es/portal/site/GRA_11748_2014/page/a6f58684-73a5-4c54-8b53-

180ccdf69557 consulta: [9 de marzo de 2015].

ANDRÉS, M.A. (2005). "Propuesta de indicadores del proceso de enseñanza/aprendizaje en la formación profesional en un contexto de gestión de calidad total" Revista Electrónica de Investigación y Evaluación Educativa, Vol. 11, №.1 doi: 10.7203/relieve.11.1.4197 http://www.uv.es/RELIEVE/v10n2/RELIEVEv11n1 4.htm [Consulta: 2 de marzo 2016].

BENNETT B., KERN J., GUDENRATH A., MCINTOSH P (2011). "The Flipped Class Revealed" The Daily Riff.<http://www.thedailyriff.com/articles/the-flipped-class-what-does-a-good-one-looklike-692.php $>$ [Consulta: 15 de marzo de 2016].

BETHANY B. S- (2012) "Flip Your Classroom to Increase Active Learning and Student Engagement"VV.AA. En 28th Annual Conference on Distance Teaching \& LearningWisconsin, EE.UU. University of Wisconsin, pp,1-5.

BOTTI V. (2014). "Comunicación. En Vicerrectorado de las Tecnologías de la Información y las Comunicaciones Valencia", UPV

ESTELLES-MIGUEL, S., ALBARRACIN GUILLEM, J.M.. y PALMER GATO, M.E. (2015). "Flip Teaching en la Asignatura de Dirección de Producción y Operaciones". Publicado en Congreso INRED 2015. pp 1-9. Doi : hrrp//dx.doi.org/10.4995/INRED2015.2015.1645.

ESTELLES-MIGUEL, S., RIUS SOROLLA, G y GIL, M. (2014). "Designing Practical Activities for Skills Development". Publicado en Innovation and Teaching Technologies-New Directions in Research, Practice and Policy. Editorial Springer. Suiza, pp 139-147.

ESTELLES-MIGUEL, S, ALBARRACIN GUILLEM, JM, PALMER GATO, ME Y PERIS-ORTIZ, M. (2016). "Aprendizaje y Evaluación basada en Proyectos en una Asignatura de Dirección de Operaciones". Publicado en IN-RED 2016 Congreso Nacional de Innovación Educativa y de Docencia en Red, pp 434-441.

OLTRA, J.V. (2015) "Diseño de unaexperiencia de Flip-Teaching para la asignaturaDeontología y Profesionalismoa impartir en la EscuelaTécnica Superior de IngenieraInformática de la UPV" INRED $2015 \quad<$ http://ocs.editorial.upv.es/index.php/INRED/INRED2015/paper/view/1646> [Consultado: 2 de abril de 2016]

ROSHAN S. (2011) "The best way to reach each student? Private school Math teacher flips learning" en The Daily Riff.< http://www.thedailyriff.com/articles/the-best-way-to-reach-each-student-privateschool-flips-learning-547.php $>$ [Consulta: 2 de mayo 2015]

SAMS A., BERGMANN J. (2013) "Flip Your Students' Learning" en Technology-Rich Learning Vol $70 \mathrm{n}^{\circ} 6$, pp. 16-20.

(c)) BY-NC-ND 2017, Universitat Politècnica de València 
TEDESCO, J.C. (2010). "La educación en el horizonte 2020". Madrid. Fundación Santillana.

UPV (2014) "Comunicado de la Universitat Politècnica de València sobre Flip Teaching” Disponible en www.upv.es/entidades/ETSINF/info/U0663284.pdf consultado 20/03/2015 consulta: [23 de marzo de 2015].

VASILEVA-STOJANOVSKA T., MALINOVSKI T., DOBRIJOVEVSKI M.V. y TRAJKOVIK V.(2015) "Impact of satisfaction, personality and learning style on educational outcomes in a blended learning environment" en Learning and Individual Differences (Article in press) $<$ do:10.1016/j.lindif.2015.01.018>[Consulta: 18 de mayo 2015].

ZABALA VIDELA, A. y ARNAU BELMONTE, L. (2007). "11 ideas clave. Cómo aprender y enseñar competencias" en Colección Ideas Clave. Serie Didáctica/Diseño y desarrollo curricular. Editorial GRAÓ de IRIF, SL. Barcelona. 